\title{
EDITORIAL
}

\section{The Positive Influences of Increasing Age at Diagnosis of Inflammatory Bowel Disease on Disease Prognostication in Asian Perspective}

\author{
Raja Affendi Raja Ali \\ Gastroenterology Unit, Department of Medicine \& Medical Molecular Biology Institute, National University of Malaysia Medical Centre, \\ Kuala Lumpur, Malaysia
}

\section{Article: Old Age at Diagnosis Is Associated With Favorable Outcomes in Korean Patients With Inflammatory Bowel Disease (Intest Res 2015;13:60-67)}

The incidence and prevalence of 'western disease'; an IBD is increasing steadily for the past 2-4 decades in Asia pacific region. ${ }^{1}$

Based on the western epidemiologic data of IBD, a bimodal age distribution for $\mathrm{CD}$ and UC is observed. An initial peak for CD and UC is in the third decade whereas the second peak is between the ages of 40 to 70 years old. ${ }^{2.3}$ IBD experts in western countries have experienced for many decades in various clinical spectrum of IBD. However, the knowledge of the influence and impact of age on the clinical course, prognostication and therapeutic achievement in patients with $\mathrm{CD}$ and UC is conflicting, limited and not well defined.

Prior studies have identified specific differences between early-onset and adult-onset CD that may indicate the earlyonset disease represents a more severe form. The French IBD database $^{4}$ had demonstrated that early-onset CD $(<16$ years old) were more likely to be considered to have active disease, higher cumulative risk of thiopurine and anti-tumour necrosis factor agent use than adult-onset $\mathrm{CD}$. The study also highlighted that despite the rate of smoking being less, early-onset CD was more active, was treated more aggressively with medications as compared to adult-onset CD patients. Quezada SM et al. ${ }^{5}$ have shown that in their CD cohort, old age patients were

Received January 2, 2014. Accepted January 5, 2014.

Correspondence to Raja Affendi Raja Ali, Gastroenterology Unit, Department of Medicine \& Medical Molecular Biology Institute, National University of Malaysia, Jalan Yaacob Latif, 56000 Cheras, Kuala Lumpur, Malaysia. Tel: +82-60-3-9145-6094, Fax: +82-60-3-9145-6692, E-mail: draffendi@ ppukm.ukm.edu.my

Financial support: None. Conflict of interest: None. more likely to have less complicated disease, but it may be confounded by differences in disease location and duration when compared with younger patients.

For the UC, Ha CY et al. ${ }^{6}$ revealed that patients with earlyonset (18-30 years old) and late-onset ( $>50$ years old) have similar initial clinical presentations, but differ in disease risk factors. The late-onset UC patients have significantly achieved steroid-free clinical remission ( 64 vs. $49 \% ; P=0.01$ ) compared to the early-onset UC patients. In contrast, several other studies found no differences or similar response in clinical course and therapeutic responsiveness between younger and older patients presented with flare of UC. ${ }^{7-9}$

With the aging of the population globally, the incidence of late-onset CD and UC is expected to be increased. Currently, IBD in patients aged $>60$ accounts for $10-15 \%$ of cases of disease. ${ }^{10}$ Therefore, careful attention on the age of diagnosis of IBD is important for optimizing the therapy of IBD.

To date, data on clinical course, therapeutic achievement and outcomes based on the Montreal Classification System in Asia are scarce. ${ }^{11}$ A retrospective large cohort study from the two tertiary referral centers in south-east Korea was used to compare clinical characteristic based on age at diagnosis according to Montreal Classification System. ${ }^{12}$ The authors found that the most common manifestation of IBD at diagnosis for old age group was haematochezia as opposed to abdominal pain for young patients. However, no difference was observed when patients were stratified into $\mathrm{CD}$ and UC. It is interesting to observe that in the sub-analysis of IBD cohort, there was no bimodal age distribution for CD cohort contrary to the western findings.

(c) Copyright 2015. Korean Association for the Study of Intestinal Diseases. All rights reserved.

This is an Open Access article distributed under the terms of the Creative Commons Attribution Non-Commercial License (http://creativecommons.org/licenses/by-nc/3.0)

which permits unrestricted non-commercial use, distribution, and reproduction in any medium, provided the original work is properly cited. 
Furthermore, there was a male predominant (in younger age group) as oppose to a traditional slight female predominance based on the western data and the most frequent location was ileal (L1) in all age group. Perianal disease was observed less frequently in the old age cohort reflecting better prognostication factor and this is similar to the recent finding in western study. ${ }^{5}$ However, there is no significant difference in the location of disease among all CD patients (but, the authors noted the small bowel disease was the most common site in all age group) which is similar to the findings by Ye et al. ${ }^{13}$ who reported previously that two-thirds of Korean CD patients had small bowel disease but differ significantly from the western data. ${ }^{14,15}$

With regards to UC, the authors have found a similar bimodal age distribution to the western data with the two peak ages in the third and sixth decades of life and there were no significant differences among the groups by gender and extent of disease based on age at diagnosis. ${ }^{12}$ Interestingly, the data for the disease extent across all age group was comparable with the previous findings by Ha et al. ${ }^{6}$ which also used the Montreal Classification system. However, several studies have shown less extensive disease when UC is diagnosed at the later life.

Overall, this retrospective and a relatively large cohort with majority of young patients with CD and old age patients with UC (might represent a true population of IBD) and a long duration follow-up from Korea have demonstrated that age at diagnosis of IBD based on the Montreal Classification System provide a valuable prognostic information for Asian IBD patients. ${ }^{12}$ It clearly shown that the remission status, cumulative rate of surgery, cumulative utilization of immunomodulators and biologics were more favorable for old age than young age patients. Furthermore, stratification of IBD by age at diagnosis in the future may provide support for the concept of genetic heterogeneity.

In conclusion, with the current treatment strategy for IBD; 'step-up, top-down or accelerated step-up' approach which is not directly applicable for the older age patients who are confounded with multiple co-morbidities and alteration of the functions such as immunologic and cardio-metabolic status and poly-pharmacy that potentially affect the clinical course of IBD. Therefore, the current treatment strategy for IBD may have to be modified to account for late-onset or older age group of IBD patients.

\section{REFERENCES}

1. Prideaux L, Kamm MA, De Cruz PP, Chan FK, Ng SC. Inflammatory bowel disease in Asia: a systematic review. J Gastroenterol Hepatol 2012;27:1266-1280.
2. Loftus CG, Loftus EV, Jr., Harmsen WS, et al. Update on the incidence and prevalence of Crohn's disease and ulcerative colitis in Olmsted County, Minnesota, 1940-2000. Inflamm Bowel Dis 2007;13:254-261.

3. Zimmerman J, Gavish D, Rachmilewitz D. Early and late onset ulcerative colitis: distinct clinical features. J Clin Gastroenterol 1985;7:492-498.

4. Pigneur B, Seksik P, Viola S, et al. Natural history of Crohn's disease: comparison between childhood- and adult-onset disease. Inflamm Bowel Dis 2010;16:953-961.

5. Quezada SM, Steinberger EK, Cross RK. Association of age at diagnosis and Crohn's disease phenotype. Age Ageing 2013;42:102-106.

6. Ha CY, Newberry RD, Stone CD, Ciorba MA. Patients with late-adult-onset ulcerative colitis have better outcomes than those with early onset disease. Clin Gastroenterol Hepatol 2010;8:682-687.

7. Triantafillidis JK, Emmanouilidis A, Pomonis E, et al. Ulcerative colitis in the elderly: clinical patterns and outcome in 51 Greek patients. J Gastroenterol 2001;36:312-316.

8. Piront P, Louis E, Latour P, Plomteux O, Belaiche J. Epidemiology of inflammatory bowel diseases in the elderly in the province of Liege. Gastroenterol Clin Biol 2002;26:157-161.

9. Weber NK, Bruining DH, Loftus EV, Jr., et al. Comparative outcomes of younger and older hospitalized patients with inflammatory bowel disease treated with corticosteroids. Inflamm Bowel Dis 2013;19:2644-2651.

10. del Val JH. Old-age inflammatory bowel disease onset: a different problem? World J Gastroenterol 2011;17:2734-2739.

11. Satsangi J, Silverberg MS, Vermeire S, Colombel JF. The Montreal classification of inflammatory bowel disease: controversies, consensus, and implications. Gut 2006;55:749-753.

12. Choi JH, Kim ES, Cho KB, et al. Old age at diagnosis is associated with favorable outcomes in Korean patients with inflammatory bowel disease. Intest Res 2015;13:60-67.

13. Ye BD, Yang SK, Cho YK, et al. Clinical features and long-term prognosis of Crohn's disease in Korea. Scand J Gastroenterol 2010;45:1178-1185.

14. Vind I, Riis L, Jess T, et al. Increasing incidences of inflammatory bowel disease and decreasing surgery rates in Copenhagen City and County, 2003-2005: a population-based study from the Danish Crohn colitis database. Am J Gastroenterol 2006;101:1274-1282.

15. Lapidus A. Crohn's disease in Stockholm County during 1990-2001: an epidemiological update. World J Gastroenterol 2006;12:75-81. 